\title{
Generation and application of a high-average- power polarized soft-x-ray laser beam
}

\author{
B. R. Benware, M. Seminario, A. L. Lecher, and J. J. Rocca \\ Department of Electrical and Computer Engineering, Colorado State University, Fort Collins, Colorado 80523
}

Yu. A. Unspenskii, A. V. Vinogradov, V. V. Kondratenko, and Yu. P. Pershing

Lebedev Physical Institute, Leninskii Prospect 53, Moscow 87545, Russia

B. Bach

Hyperfine Inc., 4946 North 63rd Street, Boulder, Colorado 80301

Received July 7, 2000

\begin{abstract}
We demonstrate the generation of a highly polarized soft-x-ray beam with a compact high-repetition-rate tabletop soft-x-ray laser. The radiation emitted by a high-average-power discharge-pumped tabletop Ne-like Ar soft-x-ray laser operating at $46.9 \mathrm{~nm}$ was polarized with a pair of $\mathrm{Si} / \mathrm{Sc}$ multilayer mirrors designed for $45^{\circ}$ operation. A degree of polarization greater than 0.96 was obtained. The polarized beam was used to characterize the efficiency of a diffraction grating. Intense polarized soft-x-ray radiation generated by compact tabletop sources has the potential to affect numerous other applications. (C) 2001 Optical Society of America OCIS codes: $140.7240,340.7470,050.2770,260.5430$.
\end{abstract}

\section{INTRODUCTION}

The generation of intense polarized soft-x-ray radiation is of significant interest for numerous applications. These include the diagnostics of materials surfaces and interfaces, the characterization of soft-x-ray optical components, and the interaction of short-wavelength radiation with atomic and molecular systems that involve anisotropy. Researchers have made extensive use of highaverage-power beams of polarized coherent shortwavelength radiation produced by undulators at modern synchrotron facilities. ${ }^{1}$ Experiments have also been conducted with polarized soft-x-ray radiation from laserproduced plasmas. $^{2}$ The development of compact tabletop sources of high-power polarized soft-x-ray radiation can be expected to have a widespread effect on applications in several fields.

Tabletop soft-x-ray lasers, which are presently capable of generating coherent radiation with high peak and average power and high energy per pulse, ${ }^{3,4}$ are excellent candidates for the generation of intense polarized shortwavelength radiation. However, to date, the generation of polarized soft-x-ray laser beams has been limited to experiments conducted at large laser facilities. ${ }^{5-9}$ Typically, soft-x-ray lasers that are based on the amplification of spontaneous emission in a plasma display a very low degree of polarization. The 19.6-nm laser line in a laserpumped Ne-like Ge amplifier was observed to be polarized parallel to the target surface with a degree of polarization of $0.53 .^{5}$ The polarization was accounted for by the anisotropy in the radiation trapping of the resonance line in an expanding plasma, which has a velocity gradient in the direction normal to the target surface. A larger degree of polarization in this line of Ne-like Ge was achieved by double-pass amplification with a half-cavity in which an oblique incidence multilayer mirror was used as a polarizer. ${ }^{6}$ A different approach in which the output of an injector plasma was linearly polarized and coupled into a Ne-like Ge amplifier produced polarized pulses of $23.2-$ and $23.6-\mathrm{nm}$ radiation with a degree of polarization of $\sim 0.98$ and a pulse energy of $\sim 20$ nJ. $^{7}$ However, the average power in all these experiments was very low because the lasers were capable of firing at a maximum repetition rate of only several shots per hour.

The recent demonstration of high-repetition-rate discharge-pumped soft-x-ray lasers ${ }^{3,10,11}$ opens the opportunity of generating high-average-power polarized soft-xray radiation with a tabletop device. Our group has recently reported the generation of a laser average power of $3.5 \mathrm{~mW}$ at $46.9 \mathrm{~nm}$ by operating a discharge-pumped collisionally excited Ne-like Ar tabletop laser at a repetition rate of $4 \mathrm{~Hz} .{ }^{10}$ The peak power of this high-repetitionrate soft-x-ray laser is approximately $0.6 \mathrm{MW}$. In this laser, which has a size similar to some of the most widely utilized visible and ultraviolet gas lasers, the gain media is an elongated plasma column generated in a gas-filled capillary channel by a fast-discharge-current pulse. The magnetic force of the current pulse rapidly compresses the plasma to form a dense and hot column with a lengthto-diameter ratio approaching 1000:1. The laser pulse is generated by single-pulse amplification of spontaneously emitted radiation in a plasma column in which a population inversion is created in the $3 s{ }^{1} P_{1}-3 p{ }^{1} P_{0}$ transition of Ne-like Ar ions by collisional electron excitation. Because of the high radial symmetry of the gain media, the laser radiation can be expected to be essentially unpolarized. However, the recent development of high- 
reflectivity multilayer mirrors at this wavelength makes possible the polarization of the beams produced by this compact tabletop source. In this paper we report the generation of intense polarized $46.9-\mathrm{nm}$ radiation by the combination of a tabletop discharge-pumped Ne-like $\mathrm{Ar}$ soft-x-ray laser and a pair of Si/Sc mirrors designed to operate at an incidence angle of $45^{\circ}$. The laser radiation was utilized to characterize the diffraction efficiency of a grating etched in single-crystal silicon. This is, to our knowledge, the first application of polarized soft-x-ray laser radiation.

\section{DESIGN AND FABRICATION OF THE POLARIZING MIRRORS}

Our polarizing mirrors for $\lambda=46.9 \mathrm{~nm}$ were constructed based on high-reflectivity Sc/Si coatings, ${ }^{12}$ which has been successfully applied recently to soft-x-ray laser-beam manipulation and focusing. ${ }^{13-16}$ To provide high polarization under reflection, the mirror reflectivity should be maximal for $s$ waves and minimal for $p$ waves. The structure of our multilayer mirrors was chosen to maximize the reflection of $s$ waves at an incidence angle of $45^{\circ}$ and consisted of 10 periods of $44.5 \mathrm{~nm}$ with a thickness ratio of $H(\mathrm{Sc}) / H(\mathrm{Si})=0.43$. The first layer deposited was a Si layer. A protective Si layer was deposited at the top of the stack. DC magnetron sputtering was used to achieve material deposition with a sufficiently low interface roughness of the order of $1.3 \mathrm{~nm} .{ }^{12,17}$ The sputtering targets used consisted of $99.5 \%$ pure Sc and a 4-in $(10-\mathrm{cm})$ diameter $n$-type $\mathrm{Si}$ wafer. The deposition rates for $\mathrm{Sc}$ and Si were held constant at $0.32 \mathrm{~nm} / \mathrm{s}$ and $0.52 \mathrm{~nm} / \mathrm{s}$, respectively. During all the deposition runs the gas used for sputtering was Ar, and the pressure in the reactor was kept at $0.43 \mathrm{~Pa}$. The multilayer period and structure were analyzed by $\operatorname{CuK} \alpha$ and $\operatorname{CuK} \beta(\lambda=0.154 \mathrm{~nm}$ and $0.179 \mathrm{~nm}$ ) x-ray reflectometry.

The reflectivity for two polarizing multilayer mirrors was measured as a function of the angle of incidence. The experimental setup used to conduct the reflectometry

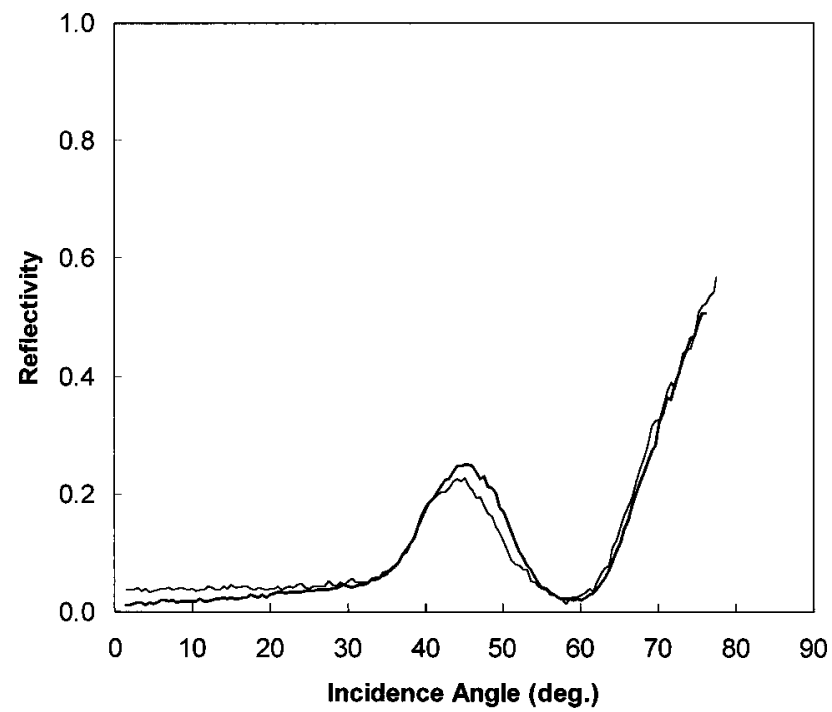

Fig. 1. Measured reflectivity of the Si/Sc multilayer mirrors at $46.9 \mathrm{~nm}$ as a function of angle of incidence. measurements as a function of angle was described previously. ${ }^{16}$ In this setup the mirror is mounted on a computer-controlled rotation stage and illuminated with $\sim 0.1$-mJ unpolarized laser pulses from a 46.9-nm Ne-like Ar capillary-discharge soft-x-ray laser operating at a repetition rate of 1 to $2 \mathrm{~Hz}$. The reflected laser signal is measured for every shot, and its intensity is normalized by a reference signal proportional to the intensity of the incoming beam. Figure 1 shows the measured reflectivity obtained as a function of angle for the two polarizers. The data show that the reflectivity of each of the two polarizing mirrors reaches a local maximum of $22 \%$ and $25 \%$ at an angle of $45^{\circ}$. At an incidence angle of $45^{\circ}$ the $s$-wave reflectivity is expected to be significantly higher than that of the $p$-wave reflectivity. Therefore the reflected beam will be partially linearly polarized.

\section{GENERATION OF A POLARIZED SOFT-X- RAY LASER BEAM}

The experimental setup that was used to polarize the laser beam is shown in Fig. 2. The vacuum chamber that housed the entire apparatus was placed at a distance of $1.5 \mathrm{~m}$ from the exit of the capillary-discharge laser. The laser pulses were generated at a repetition rate of $1 \mathrm{~Hz}$ and were polarized by a $\mathrm{Si} / \mathrm{Sc}$ multilayer-coated mirror fixed at an angle of $45^{\circ}$ from the incoming beam. To analyze the polarization of the beam, a second multilayercoated mirror, which maintained a $45^{\circ}$ angle to the reflected beam, was rotated on the axis passing through the center of both mirrors. The laser was continuously fired at a repetition rate of $1 \mathrm{~Hz}$ while the analyzer mirror was rotated from 0 to $180^{\circ}$. The intensity of the beam reflected by both mirrors was recorded with a vacuum photodiode that was mounted on a lever arm that followed the angular motion of the reflected beam. A 1-mmdiameter pinhole was placed at the entrance of the vacuum chamber to reduce the size of the beam to ensure that it could be completely captured by all of the optical components in the system. A 50\% transmissive goldplated mesh was placed before the first mirror to split a portion of the incoming beam to a second fixed vacuum photodiode that monitored the intensity of the laser beam. This reference signal was used to normalize each data point to reduce the effect of the shot-to-shot fluctuations of the laser intensity. The photodiode signals corresponding to the reflected and the reference beam intensities were recorded and stored for every laser shot with a $500-\mathrm{MHz}$ analog bandwidth, 2-gigasample/s digitizing oscilloscope (Hewlett-Packard model 54825A). Figure 3 shows the measured variation of the intensity of the laser beam after reflection on both mirrors as a function of the angle between the major axis of the polarization ellipse of the polarizer and the analyzer ( $\theta$ in Fig. 2, with $\theta=0$ when the mirrors are parallel). Each data point was normalized by its corresponding reference intensity.

Because the mirrors are not perfect polarizers, the determination of the degree of polarization requires theoretical analysis. The transformation of the beam by the pair of polarizers can be described with Stokes vectors or a Mueller matrix formalism ${ }^{18}$ as follows: 


$$
\begin{aligned}
{\left[\begin{array}{c}
I^{*} \\
M^{*} \\
C^{*} \\
S^{*}
\end{array}\right]=} & {\left[\begin{array}{c}
\text { Si/Sc mirror } \\
\text { (analyzer) }
\end{array}\right]\left[\begin{array}{c}
\text { Rotation about } \\
\text { propagation axis }
\end{array}\right] } \\
& \times\left[\begin{array}{c}
\text { Si/Sc mirror } \\
\text { (polarizer) }
\end{array}\right]\left[\begin{array}{c}
I \\
M \\
C \\
S
\end{array}\right],
\end{aligned}
$$

where $I$ is the total beam intensity, and $M, C$, and $S$ are
$C=0$, and $S=0$. After matrix manipulation, the final Stokes vector describing the beam exiting the second mirror is found to be

$$
\left[\begin{array}{c}
I^{*} \\
M^{*} \\
C^{*} \\
S^{*}
\end{array}\right]=\frac{1}{2}\left[\begin{array}{c}
\left(R_{s}{ }^{2}+R_{p}{ }^{2}\right) \cos ^{2} \theta+2 R_{s} R_{p} \sin ^{2} \theta \\
\left(R_{s}{ }^{2}-R_{p}{ }^{2}\right) \cos ^{2} \theta \\
-\sqrt{R_{s} R_{p}}\left(R_{s}-R_{p}\right) \sin 2 \theta \cos \Delta \\
-\sqrt{R_{s} R_{p}}\left(R_{s}-R_{p}\right) \sin 2 \theta \sin \Delta
\end{array}\right]
$$

The degree of polarization is

$$
p=\frac{I_{p}}{I^{*}}=\frac{\sqrt{\left[\left(R_{s}{ }^{2}-R_{p}{ }^{2}\right) \cos ^{2} \theta\right]^{2}+\left[-\sqrt{R_{s} R_{p}}\left(R_{s}-R_{p}\right) \sin 2 \theta\right]^{2}}}{\left(R_{s}{ }^{2}+R_{p}{ }^{2}\right) \cos ^{2} \theta+2 R_{s} R_{p} \sin ^{2} \theta} .
$$

the other three Stokes parameters that provide a complete description of the beam. The intensity of polarized light is given by $I_{p}{ }^{2}=M^{* 2}+C^{* 2}+S^{* 2}$. Assuming that the polarizer and analyzer mirrors are identical (this assumption is justified by the reflectivity curves shown in Fig. 1),
Fitting the expression for $I^{*}$ to the measured intensity distribution in Fig. 3, it is possible to obtain the normalized values for $R_{s}$ and $R_{p}$. To obtain $R_{s}$ and $R_{p}$, it is necessary to include the measured reflectivity of the nonpolarized beam at an incidence angle of $45^{\circ}$, where $R(45)=(1 / 2)\left(R_{s}+R_{p}\right)$. With an average value of

$\mathrm{Si} / \mathrm{Sc}$ mirror $=\frac{1}{2}\left[\begin{array}{cccc}\left(R_{s}+R_{p}\right) & \left(R_{s}-R_{p}\right) & 0 & 0 \\ \left(R_{s}-R_{p}\right) & \left(R_{s}+R_{p}\right) & 0 & 0 \\ 0 & 0 & 2 \sqrt{R_{s} R_{p}} \cos \Delta & -2 \sqrt{R_{s} R_{p}} \sin \Delta \\ 0 & 0 & 2 \sqrt{R_{s} R_{p}} \sin \Delta & 2 \sqrt{R_{s} R_{p}} \cos \Delta\end{array}\right]$,

where $R_{s, p}$ is the reflectivity for the $s$ and the $p$ waves, respectively, and $\Delta$ is the phase shift between the $s$ and the $p$ waves after reflection. The rotation matrix is given by

$$
\text { Rotation }=\left[\begin{array}{cccc}
1 & 0 & 0 & 0 \\
0 & \cos 2 \theta & \sin 2 \theta & 0 \\
0 & -\sin 2 \theta & \cos 2 \theta & 0 \\
0 & 0 & 0 & 1
\end{array}\right] .
$$

The angle $\theta$ is the same as shown in Fig. 2 and represents the angle between the major axes of the polarization ellipse for the polarizer and analyzer mirrors. The initial Stokes vector in this case describes unpolarized light with a normalized intensity and has the values $I=1, M=0$,

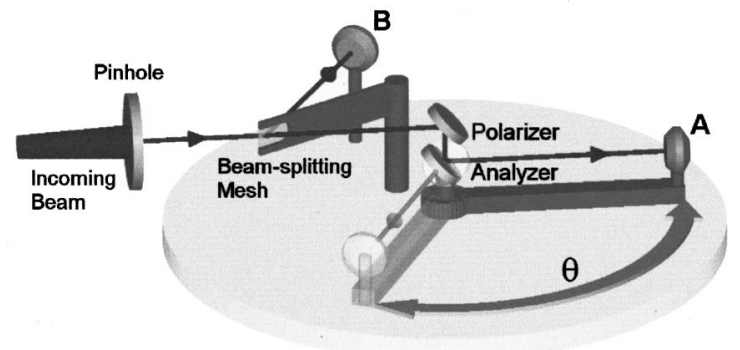

Fig. 2. Schematic representation of the setup utilized to polarize the laser beam. Labels A and B identify the fixed and the rotating vacuum photodiodes, respectively.
$R(45)=23.5 \%$ obtained from the measurements (see Fig. 1) $R_{s}$ and $R_{p}$ are determined to be 0.414 and 0.056 , respectively. With these values the maximum degree of polarization, which occurs at angles $\theta=0$ and $180^{\circ}$, is $p$ $=0.965$. This value, obtained assuming identical polarizing mirrors, represents the lower limit of the degree of polarization achieved. Considering that in reality the mirrors are similar but not identical, the maximum degree of polarization obtained is slightly higher. The remaining parameters of a polarized beam (type of polarization and direction) can also be determined by the Stokes parameters. The angle of the major axis of the polarization ellipse with respect to the $s$ direction of the second mirror is

$$
\zeta=(1 / 2) \tan ^{-1}\left(\frac{C^{*}}{M^{*}}\right)
$$

and the ratio of the major to the minor axis is

$$
\eta=\frac{I^{*}}{\left|S^{*}\right|}+\sqrt{\left(\frac{I^{*}}{\left|S^{*}\right|}\right)^{2}-1}
$$

Although it is not possible to determine $\Delta$ in $C^{*}$ and $M^{*}$ and thus $\zeta$ and $\eta$ for all angles $\theta$ with the experimental setup reported herein, at $\theta=0$ and $180^{\circ}, S^{*}=0$ and $C^{*}$ $=0$. Therefore at $\theta=0$ the beam is linearly polarized with a $96.5 \%$ degree of polarization and aligned in the $s$ 


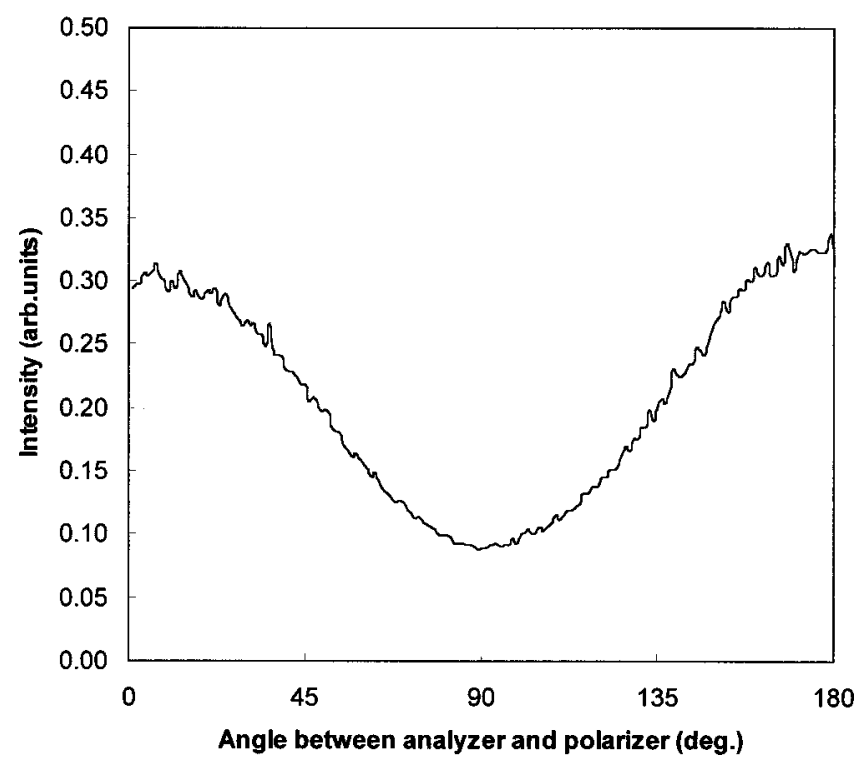

Fig. 3. Intensity of the beam after passing through the pair of polarizers as a function of the angle $\theta$ between them.

direction of the second mirror. The overall efficiency for the two-mirror polarization system at $\theta=0$ and $180^{\circ}$ is $I^{*} / I=0.5\left(R_{s}{ }^{2}+R_{p}{ }^{2}\right)=8.7 \%$. When the laser that is used in this study is optimally configured, it is possible to extract up to $\sim 0.88 \mathrm{~mJ}$ per pulse and an average power of $3.5 \mathrm{~mW}$ operating at $4 \mathrm{~Hz}^{10}$ Therefore, given the efficiency of the polarizer system described herein, it should be possible to generate an $\sim 0.3-\mathrm{mW}$ average power (76$\mu \mathrm{J} /$ pulse average at $4 \mathrm{~Hz}$ ) soft-x-ray laser beam that is better than $96 \%$ polarized. Section 4 discusses the use of this polarized beam in the characterization of the efficiency of a diffraction grating.

\section{CHARACTERIZATION OF DIFFRACTION GRATINGS}

The experimental setup utilized to characterize the diffraction efficiency of gratings for polarized 46.9-nm radiation is illustrated in Fig. 4. The measurements were conducted in the same vacuum chamber used in the polarization experiment described in Sec. 3. As in the previous case, a 1-mm-diameter pinhole was placed at the entrance of the vacuum chamber to reduce the spot size of the laser beam incident upon the grating with the purpose of measuring the diffraction efficiency at large angles of incidence. To facilitate these measurements, the grating was mounted on a rotational stage. The 46.9-nm laser radiation produced by the capillarydischarge-pumped Ne-like Ar laser was polarized utilizing the set of two $45^{\circ} \mathrm{Si} / \mathrm{Sc}$ multilayer mirrors described in the previous sections. The intensity of the beams corresponding to each of the different diffracted orders was measured with a vacuum photodiode (labeled "A" in Fig. 4) that was mounted on a rotating arm driven by a stepper motor. The data were also normalized for each pulse by the intensity of the incident laser beam for each pulse, monitored by a fixed vacuum photodiode, identified by "B" in Fig. 4. The intensity of the radiation for each of the diffraction orders was determined by operating the laser at a repetition rate of $1 \mathrm{~Hz}$ and averaging 30 consecutive laser shots. To obtain absolute measurements of the diffraction efficiency, vacuum photodiode B was placed between the second polarizer and the grating, and the ratio of the intensities between this signal and that of the reference photodiode was used for normalization. The diffraction efficiency for unpolarized radiation was also measured. In this case the polarizers were removed from the setup, and the radiation emitted by the laser was directed to impinge directly onto the grating.

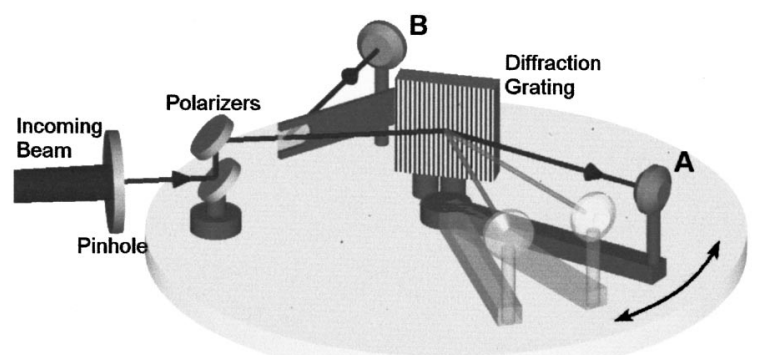

Fig. 4. Schematic representation of the experimental setup used to characterize the diffraction efficiency of a grating.
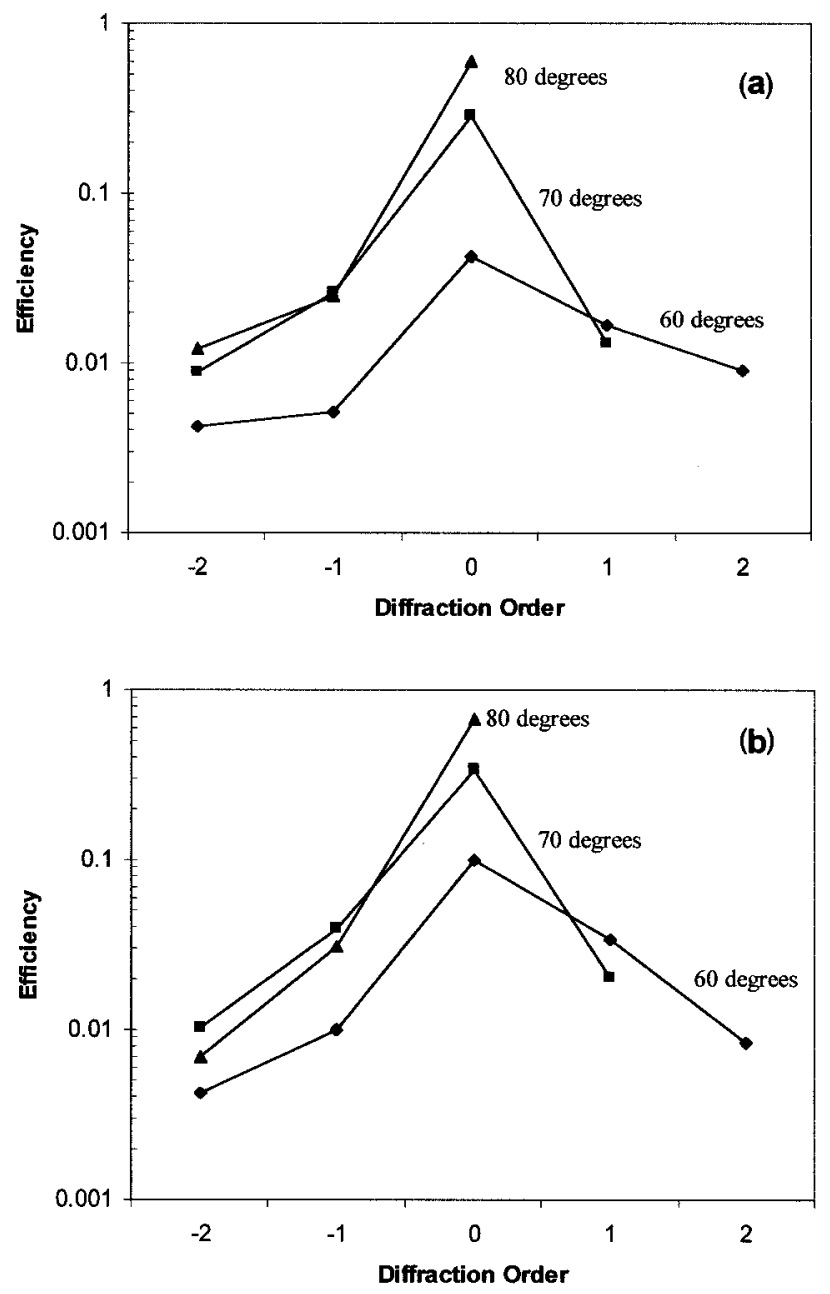

Fig. 5. (a) Diffraction efficiency of the different orders for $p$-polarized radiation at three different angles of incidence. (b) Diffraction efficiency for unpolarized radiation. 
The diffraction grating that was characterized in this experiment was prepared by ion-beam etching of singlecrystal silicon. It had a rectangular groove profile with a line density of 1200 lines $/ \mathrm{mm}$. The width of the grooves corresponded to $77 \%$ of the period, and their depth was $17.6 \mathrm{~nm}$. Figures 5(a) and 5(b) show the measured diffraction efficiency for $p$-polarization and unpolarized radiation, respectively, for incidence angles of $60^{\circ}, 70^{\circ}$, and $80^{\circ}$. Measurements were conducted for all the diffraction orders present at each of the incidence angles. The results shown are the average of at least five sets of measurements, each containing 30 laser shots for each of the diffraction orders. The error in the overall measurement was estimated to be in the range of less than $1 \%$ in the case of the zero order at an $80^{\circ}$ grazing angle of incidence for which the signal is large, to $30 \%$ for the second-order signals that have a very low intensity (often less than $1 \%$ of the impinging radiation). The diffraction efficiency at $46.9 \mathrm{~nm}$ is observed to be poor, reflecting the fact that the grating was designed for optimum operation in the range of $5-10 \mathrm{~nm}$. The intensity of the zero order dominates for all angles and increases as a function of the grazingincidence angle as a consequence of the increase of the reflectivity of the material with the angle of incidence. As expected, the first-order diffraction efficiency is larger for unpolarized radiation than for $p$-polarized radiation. The first order and minus-one order present maximum efficiency of nearly $3.5 \%$ at $60^{\circ}$ and $70^{\circ}$ incidence angles, respectively.

\section{SUMMARY AND CONCLUSIONS}

In summary, we have demonstrated a technique for the generation of high-average-power polarized soft-x-ray laser beams utilizing a compact tabletop setup. We used a pair of Si/Sc multilayer mirrors designed for operation at $45^{\circ}$ to polarize the output of a high-repetition-rate tabletop 46.9-nm laser to a degree of polarization exceeding $96 \%$. The resulting polarized beam was used to characterize the diffraction efficiency of a diffraction grating etched on a Si substrate. This is, to our knowledge, the first report of the use of a polarized soft-x-ray laser beam in an application. This intense polarized soft-x-ray laser can be expected to find important use in ellipsometry at short wavelengths and other applications.

\section{ACKNOWLEDGMENTS}

This study was supported by the National Science Foundation and by a CPOP grant from the Colorado Commission of Higher Education. We also gratefully acknowledge the support from the W. M. Keck Foundation. The development of the polarizers was the result of a collaboration sponsored by the U.S. Civilian Research and Development Foundation. The authors are grateful to I. A. Artioukov, R. M. Fechtchenko, and V. E. Levashov for helpful contributions.

\section{REFERENCES}

1. D. T. Attwood, P. Naulleau, K. A. Goldberg, E. Tejnil, C. Chang, R. Beguiristain, P. Batson, J. Bokor, E. Gullikson, M. Koibe, H. Medecki, and J. H. Underwood, "Tunable coherent radiation in the soft x-ray and extreme ultraviolet spectral region," IEEE J. Quantum Electron. 35, 709-720 (1999).

2. M. Yamamoto, K. Mayama, H. Nomura, H. Kimura, M. Yanagihara, and T. Namioka, "Soft X-ray polarization measurements with a laboratory reflectometer," Proc. SPIE 1720, 390-394 (1992).

3. J. J. Rocca, "Tabletop soft x-ray lasers," Rev. Sci. Instrum. 70, 3799-3827 (1999).

4. For example, see articles in Soft X-ray Lasers and Applications III, Proc. SPIE 3776, J. J. Rocca and L. B. Da Silva, eds. (1999).

5. T. Kawachi, K. Murai, G. Yuan, S. Ninomiya, R. Kodama, H. Daido, Y. Kato, and T. Fujimoto, "Observation of polarization of the soft x-ray laser line in neonlike germanium ions," Phys. Rev. Lett. 75, 3826-3929 (1995).

6. K. Murai, G. Yuan, R. Kodma, H. Daido, Y. Kato, M. Niibe, A. Miyake, M. Tsukamoto, Y. Fukuda, D. Neely, and A. MacPhee, "Double-pass amplification in Ge soft x-ray laser with a polarized half-cavity," Jpn. J. Appl. Phys. Lett. 33, L600-L603 (1994).

7. B. Rus, C. L. S. Lewis, G. F. Cairns, P. Dhez, P. Jaegle, M. H. Key, D. Neely, A. G. MacPhee, S. A. Ramsden, C. G. Smith, and A. Sureau, "Demonstration of amplification of a polarized soft x-ray laser beam in a neon-like germanium plasma," Phys. Rev. A 51, 2316-2327 (1995).

8. A. Sureau and P. B. Holden, "From amplification of spontaneous emission to saturation in x-ray lasers: a MaxwellBloch treatment," Phys. Rev. B 52, 3110-3125 (1995).

9. D. Benredjem, A. Sureau, B. Rus, and C. Moller, "Polarization state of the output of soft x-ray lasers through the paraxial Maxwell-Bloch approach," Phys. Rev. A 56, 51525161 (1997).

10. C. D. Macchietto, B. R. Benware, and J. J. Rocca, "Generation of millijoule-level soft x-ray laser pulses at $4-\mathrm{Hz}$ repetition rate in a highly saturated tabletop capillary discharge amplifier," Opt. Lett. 24, 1115-1117 (1998).

11. B. R. Benware, C. D. Macchietto, C. H. Moreno, and J. J. Rocca, "Demonstration of a high average power tabletop soft x-ray laser," Phys. Rev. Lett. 81, 5804-5807 (1998).

12. Yu. A. Uspenskii, V. E. Levashov, A. V. Vinogradov, A. I. Fedorenko, V. V. Kondratenko, Yu. P. Pershing, E. N. Zubalev, and V. Yu. Fedotov, "High-reflectivity multilayer mirrors for a vacuum ultraviolet interval of 35-50 nm," Opt. Lett. 23, 771-773 (1998).

13. B. R. Benware, A. Ozols, J. J. Rocca, I. A. Artiukov, V. V. Kondratenko, and A. V. Vinogradov, "Focusing on a tabletop soft x-ray laser beam and laser ablation," Opt. Lett. 24, 1714-1716 (1999).

14. J. J. Rocca, C. H. Moreno, M. C. Marconi, and K. Kanizay, "Soft x-ray laser interferometry of a plasma with a tabletop laser and a Lloyd's mirror," Opt. Lett. 24, 420-422 (1999).

15. C. H. Moreno, M. C. Marconi, K. Kanizay, J. J. Rocca, Yu. A. Uspenskii, A. V. Vinogradov, and Yu. P. Pershin, "Soft $\mathrm{x}$-ray laser interferometry of a pinch discharge using a tabletop laser," Phys. Rev. E 60, 911-917 (1999).

16. I. A. Artioukov, B. R. Benware, J. J. Rocca, M. Forsythe, Yu. A. Uspenskii, and A. V. Vinogradov, "Determination of XUV optical constants by reflectometry using a high repetition rate $46.9 \mathrm{~nm}$ laser," IEEE J. Sel. Top. Quantum Electron. 5, 1495-1501 (1999).

17. V. E. Levashov, Yu. A. Uspenskii, A. V. Vinogradov, A. I. Fedorenko, V. V. Kondratenko, Yu. P. Pershin, E. N. Zubarev, S. Mrowka, and F. Schaeffer, "Sc-Si normal incidence mirrors for a VUV interval of 35-50 nm," Int. Phys. Conf. Ser. N 159, 593 (1999).

18. D. Clarke and J. F. Granger, Polared Light in Optical Measurements (Pergamon, Elmsford, New York, 1971). 